\title{
Model-based Fault Detection, Estimation, and Prediction for a Class of Linear Distributed Parameter Systems
}

\author{
Jia Cai ${ }^{*}$, Hasan Ferdowsi and S. Jagannathan \\ * Corresponding author \\ Department of Electrical and Computer Engineering, Missouri University of Science and Technology \\ e-mail addresses: jc3k6@mst.edu (J. Cai), hfqk6@mst.edu (H. Ferdowsi), sarangap@mst.edu (S. Jagannathan)
}

\begin{abstract}
This paper addresses a new model-based fault detection, estimation, and prediction scheme for linear distributed parameter systems (DPSs) described by a class of partial differential equations (PDEs). An observer is proposed by using the PDE representation and the detection residual is generated by taking the difference between the observer and the physical system outputs. A fault is detected by comparing the residual to a predefined threshold. Subsequently, the fault function is estimated, and its parameters are tuned via a novel update law. Though state measurements are utilized initially in the parameter update law for the fault function estimation, the output and input filters in the modified observer subsequently relax this requirement. The actuator and sensor fault functions are estimated and the time to failure (TTF) is calculated with output measurements alone. Finally, the performance of detection, estimation and a prediction scheme is evaluated on a heat transfer reactor with sensor and actuator faults.
\end{abstract}

Key words: Fault detection, Fault estimation, Fault prognosis, Partial differential equations, and Distributed parameter systems.

\section{Introduction}

The design of fault detection and prediction scheme is a critical part of improving system reliability (Jiang, Marcel, and Vincent, 2006). Therefore several model-based detection and prognostics schemes have been introduced in the literature for industrial systems, which are traditionally described by ordinary differential equations (ODEs). A robust prognostic scheme was developed by Hansen, Hall, and Kurtz (1995). Fault diagnosis for gearbox was introduced by utilizing a mathematical model of the physical systems (Bartelmus, 2001). Vania and Pennacchi (2004) proposed a detection and isolation scheme by using system representation. Isermann (2004) introduced a model-based fault detection and diagnosis scheme by generating symptoms. Jiang and Chowdhury (2005) utilized an adaptive observer to handle a fault distribution function. Biswas et al. (2009) developed complementary approaches in fault detection and isolation in dynamic systems.

An adaptive threshold was generated in the research of Meseguer, Puig, and Escobet (2006) to evaluate the fault detection residual. Chinnam and Baruah (2003) and Kwan et al. (2003) developed a stochastic process model to approximate the fault and estimate the remaining useful life (RUL) or time to failure (TTF) of the system whereas the

This research is supported in part by NSF Grant IIP \#1134721 for Center on Intelligent Maintenance Systems and intelligent Systems Center.
RUL was estimated in Wang and Vachtsevanos (2001) by applying the dynamic wavelet neural network $(\mathrm{NN})$.

A variety of industrial systems including fluid flows, thermal convection and chemical reaction processes are classified as distributed parameter systems (DPS) since the system state changes with both time and space. Therefore, the ODE models given by lumped parameter representation for DPS are unsuitable to mimic their behavior (Patan and Ucinski, 2005). Instead, the state of a DPS is described by a partial differential equation (PDE).

Several fault detection and diagnosis schemes have been introduced in the literature for DPS. Christofides (2001) approximated DPS with finite dimensional ODEs; then, the reduced order ODE model was utilized in the development of fault detection and diagnosis schemes. A detection observer based on the approximate finite dimensional slow subsystem was introduced to detect and isolate faults in (Demetriou and Ito's research (2002). Baniamerian and Khorasani (2012) introduced a finite-dimensional geometric method for fault detection and isolation (FDI) of parabolic PDEs by constructing a set of residuals such that each one is only affected by a fault.

Despite these interesting results, these detection and diagnosis schemes (Demetriou and Ito, 2002; Baniamerian and Khorasani, 2012) used a finite dimensional ODE representation of DPS; consequently, they may suffer from false and missed alarms due to model reduction. In addition, the fault can change the dynamics of the overall sys- 
tem, thereby causing the reduced order model and resulting fault detection and diagnostics scheme to be inaccurate.

By contrast, this paper introduces a novel fault detection and estimation scheme by using a novel observer, which is designed directly based on PDE representation of DPS. Initially, a Luenberger-type observer was designed using healthy DPS dynamics to estimate system state and output. The estimated and measured system outputs are compared to generate the detection residual, which is shown to converge under healthy operating conditions in the absence of disturbance and uncertainty. A fault on the DPS can act as an external input to the detection residual dynamics causing the residual to increase. The fault is detected when this residual exceeds a predefined threshold.

Upon detecting a fault, an adaptive term is added to the observer to learn the fault function. Although the fault detection observer only requires the system output, the parameter update law requires the system state to be available at all positions, which is a major drawback.

Therefore, by using the linear property of the PDE representation, an input filter along with two output filters are utilized to develop a new observer, which allows the determination of a parameter update law that tunes unknown fault parameter estimation with measured system output alone. Upon detecting a fault by using the filter-based observer, the detection and estimation scheme is revisited.

With state and output availability, the detection residual and parameter estimation errors are shown to be bounded in the presence of any bounded uncertainties or disturbances while asymptotic convergence is demonstrated in the absence of these terms. In addition, with output alone the detection residual and parameter estimation errors are shown to be bounded under faults with bounded uncertainties or disturbances. Moreover, by comparing the estimated fault parameters with their failure limits, an explicit formula for online estimation of TTF or RUL is proposed.

The contributions of this paper include: a) the development of a novel model-based detection and estimation scheme by using the PDE-based detection observer with detectability conditions, b) the design of the detection, estimation and prediction scheme by using a filter-based observer, which not only requires the system output alone but also allows the estimation of actuator and sensor faults, and c) TTF prediction with outputs alone.

This paper is organized as follows. A class of linear DPS described by a parabolic PDE is introduced in Section 2. Then the detection and estimation scheme is developed in Section 3, when the state is measurable and in Section 4 with output alone. Finally, Section 5 applies the proposed scheme to a heat transfer reactor in simulations.

\section{Background and System Description}

The notations used in this paper are standard. A scalar function $v(x) \in L^{2}(0,1)$ is a square integrable on Hilbert space $L^{2}(0,1)$ with the norm defined as $\|v\|_{2}=\sqrt{\int_{0}^{1} v^{2}(x) d x}$.
Throughout the paper the norm of a function $v(x, t)$ is denoted by $\|v(t)\|$ and the norm of $\partial v(x, t) / \partial x$ is expressed as $\left\|v_{x}(t)\right\|$.

Consider a class of linear DPS expressed by the following parabolic PDE with Dirichlet actuation given by

$v_{t}(x, t)=\varepsilon v_{x x}(x, t)+\lambda v(x, t)+d(v(x, t), x, t)$

where $x$ is the space variable and $t \geq 0$ is the time variable with boundary conditions defined by

$v_{x}(0, t)=-q v(0, t), \quad v(1, t)=U(t), y(t)=v(0, t)$

where $v:[0,1] \times R^{+} \rightarrow R$ represents the distributed state of the system; $d(v(x, t), x, t)$ stands for the system uncertainty or disturbance; $U(t)$ denotes control input, $\lambda>0$ is a positive constant; $\varepsilon$ and $q$ are constant scalars; $v_{t}=\partial v / \partial t$, $v_{x}=\partial v / \partial x$ and $v_{x x}=\partial^{2} v / \partial x^{2}$ are partial derivatives of $v$ and $y(t)$ is the system output.

Assumption 1: The system uncertainty or disturbance is bounded above such that $\|d(v, x, t)\| \leq \bar{d}$ for all $(v, x)$ and $t \geq 0$, where $\bar{d}>0$ is a known constant. A more specific representation can be found in (Baniamerian and Khorasani, 2012; Yao and El-Farra, 2011).

In this paper, an actuator and sensor fault type at the boundary condition are considered and will be described next.

\subsection{Actuator fault}

Under a multiplicative actuator fault at the boundary condition of the DPS, the system in (1) and (2) can be described by

$v_{t}(x, t)=\varepsilon v_{x x}(x, t)+\lambda v(x, t)+d(v(x, t), x, t)$,

subject to the boundary conditions given by

$v_{x}(0, t)=-q v(0, t), v(1, t)=\theta U(t), y(t)=v(0, t)$,

where $\theta$ is the multiplicative fault parameter bounded by $|\theta| \leq \theta_{\max }$. Alternatively, the boundary condition with the actuator fault can be expressed as $v(1, t)=U(t)+h(U(t), t)$, where $h(U(t), t)=\Theta U(t)=(\theta-1) U(t)$ and $\Theta=\theta-1$.

Moreover, the fault function can be written as

$$
h(U(t), t)=\Omega\left(t-t_{i}\right) \Theta U(t),
$$

where $t_{i}$ is the time of fault occurrence and $\Omega\left(t-t_{i}\right)$ is the time profile of the fault defined by $\Omega(\tau)=\left\{\begin{array}{ll}0 & \text {, if } \tau<0 \\ 1-e^{-\kappa \tau}, & \text { if } \tau \geq 0\end{array}\right.$, where $\kappa$ represents the fault growth rate, which should be a constant. This time profile allows both incipient and abrupt faults with different growth rates $\kappa$ to be represented. However, for fault prediction, incipient faults are considered. 


\subsection{Sensor fault}

Under a sensor fault, the system measured output is written as

$y(t)=\theta_{s} v(0)$,

where $\theta_{s}$ is a positive scalar representing a multiplicative sensor fault bounded by $\theta_{s \min } \leq\left|\theta_{s}\right| \leq \theta_{s \max }$. Under healthy conditions, the value of $\theta_{s}$ is taken as unity whereas it changes in the presence of a sensor fault. The following standard assumptions are required in order to proceed.

Assumption 2: There exists a stabilizing controller that guarantees the boundedness of the system state under healthy operating conditions.

Remark 1: This assumption separates a fault with instability of the system. For fault detection, the closed-loop DPS should be stable. Smyshlyaev and Krstic (2004) proposed a state and output feedback controller by using the backstepping approach to stabilize the parabolic PDE by using a control input which is a function of output $y(t)$.

Assumption 3: The fault type is known. Moreover, a single fault occurs on the system at any given time.

Remark 2: This assumption is used for fault estimation.

Before presenting the case where only the output is available, initially the system state and output are considered available over the entire range of space so that an actuator fault can be estimated. Next when the system output alone is available, the fault detection observer is redesigned using input and output filters. Fault estimation and prognosis are performed for both actuator and sensor faults. The next section investigates the former scenario and the latter is discussed in Section 4.

\section{Detection and Estimation with State Measurements}

In this section, the system is considered initially without any disturbance and uncertainty, i.e., $d(v, x, t)=0$. An observer acting as a model is used to estimate the system state and output by utilizing DPS dynamics in healthy conditions. Fig. 1 shows that under healthy conditions with no disturbances and uncertainties, and through the selection of observer gains, the estimated output will converge to measured value and thus the detection residual, which is defined as the difference between the estimated and the measured outputs, will converge to zero. During an actuator fault, the control input applied to the original system will be different than that of the observer. Thus, the measured output will deviate from the estimated output and lead to an increase in the residual (Gertler, 2005).

A sensor fault, on the other hand, will cause a change in the measured output, which will change the estimated output through feedback control input. Thus, a sensor fault can be detected as it leads to changes in the measured and estimated outputs differently causing the detection residual to increase over a threshold.
Remark 3: In this paper, the measurements are considered noise free. In addition, in the presence of bounded uncertainties and disturbances, the detection residual is shown to be bounded instead of converging to zero.

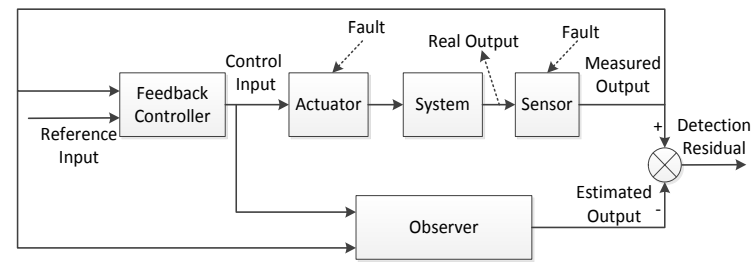

Fig. 1. Architecture of fault detection scheme.

\subsection{Fault Detection Observer}

An observer generates the state of the DPS and is tuned by the output. By using (Smyshlyaev and Krstic, 2010), define the fault detection observer as

$$
\begin{aligned}
& \hat{v}_{t}(x, t)=\varepsilon \hat{v}_{x x}(x, t)+\lambda \hat{v}(x, t)+p_{1}(x)(y-\hat{y}), \\
& \hat{v}_{x}(0, t)=-q v(0, t)+p_{10}(y-\hat{y}), \quad \hat{v}(1, t)=U(t), \\
& \hat{y}(t)=\hat{v}(0, t), \quad e(t)=y(t)-\hat{y}(t)
\end{aligned}
$$

where $\hat{v}(x, t)$ is the estimated system state, $\hat{y}$ represents estimated output, $p_{1}(x)$ and $p_{10}$ denote observer gains, and $e(t)$ is the detection residual.

Note that the fault detection observer is constructed using measured output. However, it will be observed later that when an adaptive term is incorporated into this observer to estimate fault function upon detection, the parameter tuning law will require the system state to be available.

To move on, define a state residual or state estimation error as $\tilde{v}=v-\hat{v}$ so as to analyze the performance of the observer. The state residual can also be used for fault detection in this section due to the availability of the system state; however, this is not necessary since the linear PDE in (3) transfers the effect of actuator fault to the output; thus, fault can be detected by using the output residual $e$. In the absence of disturbances and uncertainties, $d(v, x, t)=0$, the state residual dynamics without a fault is represented as

$$
\begin{aligned}
& \tilde{v}_{t}(x, t)=\varepsilon \tilde{v}_{x x}(x, t)+\lambda \tilde{v}(x, t)-p_{1}(x) \tilde{v}(0, t), \\
& \tilde{v}_{x}(0, t)=-p_{10} \tilde{v}(0, t), \quad \tilde{v}(1, t)=0 .
\end{aligned}
$$

Next the following Lemma is needed in order to proceed.

Lemma 1 (Krstic and Smyshlyaev, 2008): Consider the Volterra integral transformation

$$
\tilde{v}(x, t)=\xi(x, t)-\int_{0}^{x} L(x, \tau) \xi(\tau, t) d \tau,
$$

where

$$
L(x, \tau)=-\frac{(\lambda+b)(1-x)}{\varepsilon} \sum_{n=0}^{\infty} \frac{\left[\frac{(\lambda+b)}{\varepsilon}(2-x-\tau)(x-\tau)\right]^{n}}{2^{2 n+1} n !(n+1) !}
$$

is the solution to the hyperbolic PDE given by

$\varepsilon L_{x x}(x, \tau)-\varepsilon L_{\tau \tau}(x, \tau)=-(\lambda+b) L(x, \tau)$,

$L(1, \tau)=0, \quad L(x, x)=(\lambda+b)(x-1) /(2 \varepsilon)$.

Select observer gains $p_{1}(x)$ and $p_{10}$ as 


$$
p_{1}(x)=\varepsilon L_{\tau}(x, 0), \quad p_{10}=L(0,0) \text {. }
$$

For the sake of eliminating the unstable term $\lambda \tilde{v}(x, t)$ in (9), the transformation (11) with observer gains (15) converts the state residual dynamics in (9) and (10) to

$$
\begin{aligned}
& \xi_{t}(x, t)=\varepsilon \xi_{x x}(x, t)-b \xi(x, t), \\
& \xi_{x}(0, t)=0, \quad \xi(1, t)=0,
\end{aligned}
$$

where $b \geq 0$ is a constant that defines the convergence rate.

Next, the following lemma will show that the transformation (11) is invertible.

Lemma 2: The inverse transformation of (11) is given by

$$
\xi(x, t)=\tilde{v}(x, t)+\int_{0}^{x} M(x, \eta) \tilde{v}(\eta, t) d \eta
$$

where

$$
M(x, \eta)=-\frac{(\lambda+b)(1-x)}{\varepsilon} \sum_{n=0}^{\infty} \frac{(-1)^{n}\left[\frac{(\lambda+b)}{\varepsilon}(2-x-\eta)(x-\eta)\right]^{n}}{2^{2 n+1} n !(n+1) !}
$$

is the solution obtained through successive approximation (Krstic and Smyshlyaev, 2008) to the following hyperbolic PDE given by

$$
M_{x x}(x, \eta)-M_{\eta \eta}(x, \eta)=(\lambda+b) M(x, \eta) / \varepsilon,
$$$$
M(x, x)=(\lambda+b)(x-1) / 2 \varepsilon, \quad M(1, \eta)=0 .
$$

Proof: Follow steps in Krstic and Smyshlyaev (2008).

It will be shown that with the new observer presented herein, the detection residual converges to zero asymptotically under healthy conditions without any bounded disturbances or uncertainties and will remain ultimately bounded (UB) with them. A fault is detected by comparing the detection residual $e(t)$ with a predefined threshold $\rho$. The threshold is selected by using both the initial conditions and the bound on any system uncertainty or disturbances. The following theorem demonstrates the stability of detection residual under healthy conditions and provides fault detectability conditions.

Theorem 1 (Fault detection observer performance): Let the observer given in (7) and (8) be used to monitor the DPS defined by (3), (4) and (6). Then the state estimation error $\tilde{v}$ and the output detection residual $e(t)$ will converge to zero asymptotically under healthy operating conditions. In addition, a fault is declared active when the output detection residual $e(t)$ crosses the detection threshold $\rho$. A fault initiated at time $t_{i}$ is detectable if there exists a time $T \geq t_{i}$ and a positive constant $\mathrm{H}>\rho$, such that

$$
\begin{aligned}
& \text { i. }|h(U(T), \mathrm{T})|=H \text { and }|h(U(t), t)| \geq H \text { for } t>T \text { in } \\
& \text { the case of an actuator fault, or } \\
& \text { ii. }\left|\left(\theta_{s}-1\right) U(\mathrm{~T})\right|=H \text { and }\left|\left(\theta_{s}-1\right) U(t)\right| \geq H \text { for } t>T
\end{aligned}
$$
in the case of a sensor fault.

Proof: It is already known that the transformation (11) can map the state residual dynamics into the target system of (16) and (17) if $p_{1}(x)$ and $p_{10}$ are defined by (15). The stability of the residual dynamics can be concluded from the stability of the target system given by (16) and (17) due to the transformation made possible by (11) (Balogh and Krstic, 2002). To discuss the stability of the PDE described in (16) with boundary conditions given by (17), one must select a positive definite Lyapunov function candidate, which is half of the squared Sobolev norm of the spatial profile defined in a Hilbert space $H_{1}(0,1)$ as per Krstic and Smyshlyaev (2008)

$$
\begin{aligned}
V(t) & =\|\xi(t)\|^{2} / 2+\left\|\xi_{x}(t)\right\|^{2} / 2 \\
& =\int_{0}^{1} \xi^{2}(x, t) d x / 2+\int_{0}^{1} \xi_{x}^{2}(x, t) d x / 2
\end{aligned}
$$

The derivative of (22) is given by

$$
\dot{V}(t)=\int_{0}^{1} \xi(x, t) \xi_{t}(x, t) d x+\int_{0}^{1} \xi_{x}(x, t) \xi_{t x}(x, t) d x .
$$

By using (16) and (17) in the equation above and applying both integration by parts and Poincare inequality (Hardy, Littlewood and Polya, 1952), $\dot{V}(t)$ becomes

$$
\dot{V}(t) \leq-\pi^{2} \varepsilon\left[\int_{0}^{1} \xi^{2}(x, t) d x+\int_{0}^{1} \xi_{x}^{2}(x, t) d x\right] / 4=-\pi^{2} \varepsilon V(t) / 2,
$$

which is exponentially converging. It further yields that $\|\xi(t)\|^{2}+\left\|\xi_{x}(t)\right\|^{2} \leq e^{-\frac{\varepsilon \pi^{2}}{2} t}\left(\|\xi(0)\|^{2}+\left\|\xi_{x}(0)\right\|^{2}\right)$.

Therefore, the system defined by (16) and (17) is exponentially stable in $H_{1}(0,1)$. By using Agmon's inequality (Smyshlyaev and Krstic, 2010) we get

$$
\begin{aligned}
& \max _{x \in[0,1]}|\xi(x, t)|^{2} \leq 2\|\xi(t)\|\left\|\xi_{x}(t)\right\| \\
& \leq\|\xi(t)\|^{2}+\left\|\xi_{x}(t)\right\|^{2} \leq e^{-\frac{\varepsilon \pi^{2}}{2} t}\left(\|\xi(0)\|^{2}+\left\|\xi_{x}(0)\right\|^{2}\right)
\end{aligned}
$$

which implies that $\xi(x, t)$ converges to zero asymptotically for all $x \in[0,1]$. By using the relationship between the detection residual $\tilde{v}(x, t)$ and $\xi(x, t)$ from $(11)$, we can conclude that as $\xi(x, t) \rightarrow 0$ asymptotically, state residual $\tilde{v}(x, t) \rightarrow 0$ during healthy operating conditions.

To determine detectability conditions, an actuator fault is considered first. When an actuator fault occurs at $t_{i}$ then the state estimation error dynamics described by (9) is subject to following boundary conditions given by

$$
\tilde{v}_{x}(0, t)=-p_{10} \tilde{v}(0, t), \quad \tilde{v}(1, t)=h(U(t), t) .
$$

Applying the transformation (11) on (9) and (24) leads to (16) with boundary conditions given by

$$
\xi_{x}(0, t)=0, \quad \xi(1, t)=h(U(t), t),
$$

for $t \geq t_{i}$. Because $\tilde{v}(1, t)=\xi(1, t)-\int_{0}^{1} L(1, \tau) \xi(\tau, t) d \tau$ and $L(1, \tau)=0$ for all $0 \leq \tau \leq 1$, we can get $\tilde{v}(1, t)=\xi(1, t)$. Notice that $b=0$ before fault detection and $b>0$ after fault detection. Now a bounded state variable $\phi(x, t)$ is introduced which is defined by (16) with $b=0$ $\phi_{t}(x, t)=\varepsilon \phi_{x x}(x, t)$ subject to $\phi_{x}(0, t)=0$ and $\phi(1, t)=H$ and $\phi(x, T)=\xi(x, T)$. If $|h(U(t), t)| \geq H \quad$ for $t \geq T$ then $|\xi(x, t)| \geq \phi(x, t)$ for $0 \leq x \leq 1$ and $t \geq T$. Because 
$\bar{\phi}(x, t)=\phi(x, t)-H$ satisfies the following exponentially stable PDE given by $\bar{\phi}_{t}(x, t)=\varepsilon \bar{\phi}_{x x}(x, t), \bar{\phi}_{x}(0, t)=0$, $\bar{\phi}(1, t)=0$, the new state variable $\phi(\mathrm{x}, \mathrm{t})$ converges to $H$. Hence, for any $\omega>0$, there exists a time $t_{d} \geq T$, such that $\phi\left(0, t_{d}\right) \geq H-\omega$. Therefore, for any $\omega>0$, there exists a time $t_{d} \geq T$ such that $\left|e\left(t_{d}\right)\right|=\left|\xi\left(0, t_{d}\right)\right| \geq \phi\left(0, t_{d}\right) \geq H-\omega$. Because $H>\rho$ and if $\omega$ is selected as $\omega<H-\rho$, then $\left|e\left(t_{d}\right)\right|>\rho$ for some $t_{d} \geq T$, so that the detection of an actuator fault is guaranteed when the detectability condition in Theorem 1 is satisfied.

In the case of a sensor fault, the detection residual can be written as $e(t)=y(t)-\hat{y}(t)=\theta_{s} v(0, t)-\hat{v}(0, t)$. If we define a new distributed variable $\Delta(x, t)=\theta_{s} v(x, t)-\hat{v}(x, t)$, then the detection residual can be expressed as $e(t)=\Delta(0, t)$. By using the definition of $\Delta$ and the observer dynamics in (7) and (8), it can be shown that $\Delta$ satisfies the PDE given by $\Delta_{t}(x, t)=\varepsilon \Delta_{x x}(x, t)+\lambda \Delta(x, t)-p_{1}(x) \Delta(0, t)$, $\Delta_{x}(0, t)=-p_{10} \Delta(0, t), \quad \Delta(1, t)=\left(\theta_{s}-1\right) U(t)$, for $0 \leq x \leq 1$ and $t \geq t_{i}$. If $\tilde{v}$ in transformation (11) is replaced by $\Delta$, applying this transformation to the above PDE will lead to

$\xi_{t}(x, t)=\varepsilon \xi_{x x}(x, t)-b \xi(x, t)$, $\xi_{x}(0, t)=0, \quad \xi(1, t)=\left(\theta_{s}-1\right) U(t)$,

which is exactly the same as (16) and (25) except that the term $h(U(t), t)$ is replaced by $\left(\theta_{s}-1\right) U(t)$. Thus, by using the same steps taken in the case of an actuator fault, it can be shown that if $\left|\left(\theta_{s}-1\right) U(t)\right| \geq H$ for $t \geq T$, then for any $\omega>0$, there exists a time $t_{d} \geq T$ such that $\left|e\left(t_{d}\right)\right|=\left|\Delta\left(0, t_{d}\right)\right| \geq H-\omega$. Selecting $\omega<H-\rho$, results in $\left|e\left(t_{d}\right)\right|>\rho$ which declares the presence of a fault.

Remark 4: In the presence of bounded uncertainties or disturbances, the dynamics of the observer error becomes $\tilde{v}_{t}(x, t)=\varepsilon \tilde{v}_{x x}(x, t)+\lambda \tilde{v}(x, t)-p_{1}(x) \tilde{v}(0, t)+d(v, x, t)$ with boundary condition (10). By applying inverse transformation (18) to these dynamics, you get $\xi_{t}(x, t)=$

$$
\varepsilon \xi_{x x}(x, t)-b \xi(x, t)+d(v, x, t)+\int_{0}^{x} d(v, \eta, t) M(x, \eta) d \eta
$$

and (17). Now select (22), and it can be shown that with $b=0,\|\xi(t)\| \leq \frac{2 \sqrt{4+\pi^{2}}}{\varepsilon \pi^{2}} d_{M}$ and $\left\|\xi_{x}(t)\right\| \leq \frac{2 \sqrt{4+\pi^{2}}}{\varepsilon \pi^{2}} d_{M}$ with $d_{M}=(1+\bar{m}) \bar{d} \quad$ and $\bar{m}=\max _{0 \leq x, \eta \leq 1}|M(x, \eta)|$.

Remark 5: By using Agmon's inequality and the results of Remark 4, the detection residual can be expressed as $|e(t)|=|\xi(0, t)| \leq \frac{2 \sqrt{2\left(4+\pi^{2}\right)}}{\varepsilon \pi^{2}} d_{M}(1+\bar{m}) \bar{d}$. To detect the fault, a predefined threshold must be modified as $\rho=\rho_{0}+k_{m} \bar{d}$ where $\rho_{0}$ is the threshold without uncertainties and disturbances, and $k_{m}=\sqrt{\frac{2 \sqrt{2}(\varepsilon+2 b)}{\sqrt{\varepsilon(\varepsilon+b)} \varepsilon b \pi^{2}}}(1+\bar{m})$. Note the newly defined threshold is greater than the one without uncertainties and disturbances.

\subsection{Fault Estimation}

Upon detection, the fault parameter has to be estimated. Although, both actuator and sensor faults are detectable by the proposed detection observer, the current method does not allow estimation of sensor fault function, since the dynamics of observer and residual change due to a sensor fault, makes the transformation (11) inadmissible. Therefore, in this section, the fault estimation is performed for actuator faults only.

An adaptive estimator, which is only activated upon detection, is added to the boundary condition of the observer (7) and (8) as (7) with boundary conditions

$$
\begin{aligned}
& \hat{v}_{x}(0, t)=-q v(0, t)+p_{10} e(t), \\
& \hat{v}(1, t)=U(t)+\hat{h}(U(t) ; \hat{\Theta}(t)), \hat{y}(t)=\hat{v}(0, t),
\end{aligned}
$$

where $\hat{h}$ is the estimated fault dynamics given by the adaptive estimator

$$
\hat{h}(U(t), \hat{\Theta}(t))=\hat{\Theta}(t) U(t),
$$

with $\hat{\Theta}(t)$ as the estimated fault parameter where $\hat{\Theta}(0)=0$.

By taking the difference between the observer dynamics in (7), (26), and (27) and the actual system dynamics in (3) and (4) and applying Assumption 2, the state residual dynamics upon detecting an actuator fault can be expressed as (9) subject to

$$
\tilde{v}_{x}(0, t)=-p_{10} e(t), \tilde{v}(1, t)=\tilde{\Theta}(t) U(t),
$$

where $\tilde{\Theta}(t)=\Theta-\hat{\Theta}(t)$ is the fault parameter estimation error. Next the performance of the observer is discussed in the presence of an actuator fault.

Theorem 2 (Performance of an actuator fault estimation): Let the boundary condition of the observer in (8) be modified using (26) and (27) in order to estimate the state and output of the system defined in (3) and (4). In the presence of an actuator fault, consider the parameter tuning law

$$
\begin{aligned}
\dot{\hat{\Theta}}(t)= & \beta \varepsilon U(t)\left(\tilde{v}_{x}(1, t)+\int_{0}^{1} M_{x}(1, \eta) \tilde{v}(\eta) d \eta\right) \\
& -\hat{\Theta}(t) \gamma\left\|\tilde{v}(x, t)+\int_{0}^{x} M(x, \eta) \tilde{v}(\eta, t) d \eta\right\|^{2},
\end{aligned}
$$

for fault estimation where $\beta>0$ is the adaptation rate, $0<\gamma<2 \beta b / \Theta_{\max }^{2}$ is the stabilizing term, and $M(x, \eta)$ is 
given by (19), then the state residual converges to zero and the parameter estimation error is bounded.

Proof: First apply transformation (11) on the residual dynamics (9) and (29) to get PDE (16) with boundary conditions given by

$$
\xi_{x}(0, t)=0, \quad \xi(1, t)=\tilde{\Theta}(t) U(t) .
$$

Now select the Lyapunov function candidate

$V(t)=\int_{0}^{1} \xi^{2}(x, t) d x / 2+\tilde{\Theta}^{2}(t) /(2 \beta)$,

whose first derivative is given by $\dot{V}(t)=\int_{0}^{1} \xi(x, t) \xi_{t}(x, t) d x+\tilde{\Theta}(t) \dot{\tilde{\Theta}}(t) / \beta$. By substituting (16) in the first derivative, we will arrive at

$$
\dot{V}(t)=\varepsilon \int_{0}^{1} \xi(x, t) \xi_{x x}(x, t) d x-b \int_{0}^{1} \xi^{2}(x, t) d x+\tilde{\Theta}(t) \dot{\tilde{\Theta}}(t) / \beta
$$

Applying integration by parts and using boundary conditions given by (31) will lead to

$$
\begin{array}{r}
\dot{V}(t)=\varepsilon \xi_{x}(1, t) \tilde{\Theta}(t) U(t)-\varepsilon \int_{0}^{1} \xi_{x}^{2}(x, t) d x \\
-b \int_{0}^{1} \xi^{2}(x, t) d x+\tilde{\Theta}(t) \dot{\tilde{\Theta}}(t) / \beta
\end{array}
$$

To represent this update law in terms of a transformed system state, instead of the actual system state, the inverse transformation (18) and its first derivative with respect to $x$ given by $\xi_{x}(1, t)=\tilde{v}_{x}(1, t)+\int_{0}^{1} M_{x}(1, \eta) \tilde{v}(\eta, t) d \eta$ will be utilized in (30) to get

$$
\dot{\hat{\Theta}}(t)=\beta \varepsilon U(t) \xi_{x}(1, t)-\hat{\Theta}(t) \gamma\|\xi(t)\|^{2} .
$$

Equation (34) eliminates the positive term $\varepsilon \xi_{x}(1, t) \tilde{\Theta}(t) U(t)$.

By applying the proposed parameter update law in the derivative of the Lyapunov function candidate, we get

$$
\begin{gathered}
\dot{V}(t)=-\varepsilon \int_{0}^{1} \xi_{x}^{2}(x, t) d x-b\|\xi(t)\|^{2}+\tilde{\Theta}(t) \hat{\Theta}(t) \gamma\|\xi(t)\|^{2} / \beta \\
\leq-b\|\xi(t)\|^{2}-\gamma\|\xi(t)\|^{2}\left[\tilde{\Theta}^{2}(t)-\Theta^{2}{ }_{\text {max }}\right] / 2 \beta \\
\leq-\left(2 \beta b-\Theta^{2}{ }_{\text {max }} \gamma\right)\|\xi(t)\|^{2} /(2 \beta)-\gamma \tilde{\Theta}^{2}(t)\|\xi(t)\|^{2} /(2 \beta)
\end{gathered} .
$$

Thus, when $\gamma<2 \beta b / \Theta_{\max }^{2}, \dot{V} \leq 0$ and $\|\xi(t)\|$ and $|\tilde{\Theta}|$ are bounded. Now, define $S=\{\|\xi(t)\|, \tilde{\Theta}(t) \mid \dot{V}(\|\xi(t)\|, \tilde{\Theta}(t))=0\}$, when $\dot{V}=0$. Since the largest invariant set contained in $\mathrm{S}$, is same as $\mathrm{S}$, the asymptotic convergence of $\|\xi(t)\|$ to zero and boundedness of $|\tilde{\Theta}|$ can be shown by using LaSalle's theorem (LaSalle, J. and Lefschetz, S., 1961).

Remark 6: In the presence of $d(v, x, t)$, it can be shown that with $(30)\|\xi(t)\| \leq \sqrt{\beta /\left[b\left(\beta b-\Theta^{2}{ }_{\text {max }} \gamma\right)\right]}(1+\bar{m}) \bar{d}$. Therefore, $\xi(x, t)$ is bounded for $x \in[0,1]$. From (31), notice that $\xi(1, t)=\tilde{\Theta}(t) U(t)$, so that $\tilde{\Theta}(t)$ is also bounded.

\section{Estimation and Prediction with Outputs}

In this case, only the system output is considered measurable without the system state being available. The detec- tion observer had to be redesigned and its tuning law had to be carefully selected.

\subsection{Filter-Based Observer}

In the case of the redesign of the filter-base observer, the boundary value $v(0, t)$ is available. The following steps have to be taken: (a) Convert the system dynamics to an observable form. (b) Design a filter-based observer based on known information, namely the control input and the measured output. (c) Prove the stability of the observer under healthy conditions. (d) Incorporate adaptive terms in the observer to estimate unknown fault parameters, upon detection of a fault.

In the first step, the system plant $(3,(4)$ and (6) is converted to an observable form by utilizing the transformation (Smyshlyaev and Krstic, 2004)

$$
z(x, t)=v(x, t)-\int_{0}^{x} l(x, \tau) v(\tau, t) d \tau,
$$

where $z(x, t)$ is the new state variable of the system in the observable form and $l(x, \eta)$ is the solution of the hyperbolic $\operatorname{PDE} \quad l_{x x}-l_{\eta \eta}=\lambda l(x, \eta) / \varepsilon \quad, \quad l(1, \eta)=0 \quad$ and $l(x, x)=\lambda(1-x) / 2 \varepsilon$. Therefore, this transformation can convert the system (3), (4) and (6) in the presence of an actuator and a sensor, both of which fault respectively into

$$
\begin{aligned}
z_{t}(x, t) & =\varepsilon z_{x x}(x, t)-\varepsilon\left[l_{\eta}(x, 0)+q l(x, 0)\right] z(0, t) \\
& +d(v(x), x, t)-\int_{0}^{x} d(v(\tau), \tau, t) l(x, \tau) d \tau, \\
z_{x}(0, t) & =-(\lambda / 2 \varepsilon+q) z(0, t), \quad z(1, t)=\theta U(t),
\end{aligned}
$$

and

$y(t)=\theta_{s} z(0, t)$.

Note that $v(0, t)=z(0, t)$. Under healthy conditions where $\theta=\theta_{s}=1$, consider linear DPS in (36) and (37) with $U(t)$ $-\varepsilon\left(l_{\eta}(x, 0)+q l(x, 0)\right) v(0, t)$ and $-(\lambda / 2 \varepsilon+q) v(0, t)$ as external inputs (Krstic and Smyshlyaev, 2008). By using the superposition principle, its solution can be expressed by summing the response of the PDE due to each external input. Therefore, $z(x, t)$ can be expressed as a combination of the solutions defined by Krstic and Smyshlyaev (2008) $\Lambda_{t}(x, t)=\varepsilon \Lambda_{x x}(x, t), \Lambda_{x}(0, t)=0, \quad \Lambda(1, t)=U(t)$, where $\Lambda(x, t)$ is referred to as an input filter since it is derived from the input of the actual system $U(t)$ (Smyshlyaev and Krstic, 2008). Next consider

$$
A_{t}(x, t)=\varepsilon A_{x x}(x, t), \quad A_{x}(0, t)=y(t), \quad A(1, t)=0,
$$

where $A(x, t)$ is an output filter since it is derived from output of the actual system $y(t)$. In addition, consider

$$
\begin{gathered}
B_{t}(x, \eta, t)=\varepsilon B_{x x}(x, \eta, t)+\delta(x-\eta) y(t) \\
B_{x}(0, \eta, t)=0, \quad B(1, \eta, t)=0
\end{gathered}
$$

where $B(x, \eta, t)$ is another output filter. 
Define the observer in terms of the new state variable as $\hat{z}(x, t)=\Lambda(x, t)+[-\lambda /(2 \varepsilon)-q] \mathrm{A}(x, t)$

$+\int_{0}^{1} \varepsilon\left[-l_{\eta}(s, 0)-q l(s, 0)\right] \mathrm{B}(x, s, t) d s, \quad \hat{y}(t)=\hat{z}(0, t)$,

where $\hat{z}(x, t)$ is an estimate of $z(x, t)$ and $\hat{y}(t)$ is an estimate of $y(t)$ under healthy operating conditions.

It is shown in the next theorem that the observer state estimation error $\tilde{z}(x, t)=z(x, t)-\hat{z}(x, t)$ is ultimately bounded under healthy conditions with $\theta=\theta_{s}=1$. To perform fault detection, based on the observer error, one must define the detection residual $\bar{e}(t)=y(t)-\hat{y}(t)$ since the only available measurement is $y(t)$. A fault is detected when the detection residual $\bar{e}(t)$ exceeds the predefined detection threshold $\rho$. Next, the fault detectability condition is introduced in the following theorem.

Theorem 3 (Output-based fault detection observer performance): Let the observer in (39) be used to monitor the DPS defined by (36)-(38) with bounded disturbances. Then the state estimation error $\tilde{z}$ and detection residual $\bar{e}(t)$ are bounded under healthy operating conditions. Further, a fault initiated at time $t_{i}$ is detectable if there exists a time $T \geq t_{i}$ and a positive constant $H$, such that

i. $|(\theta-1) U(t)| \geq H$ for $t \geq T$ where $H>\rho+k_{l} \bar{d} \quad$ in the case of an actuator fault, or

ii. $\left|\left(\theta_{s}-1\right) U(t)\right| \geq H$ for $t \geq T$ where $H>\rho+k_{l} \bar{d} \theta_{s \max }$ in the case of a sensor fault.

where $k_{l}=2 \sqrt{2\left(\pi^{2}+4\right)}(1+\bar{l}) /\left(\varepsilon \pi^{2}\right)$ and $\bar{l}=\max _{0 \leq x, \eta \leq 1} l(x, \eta)$.

Proof: During healthy conditions with $\theta=\theta_{s}=1$, the state residual satisfies the stable PDE given by

$$
\begin{gathered}
\tilde{z}_{t}(x, t)=\varepsilon \tilde{z}_{x x}(x, t)+d(v, x, t)-\int_{0}^{x} d(v, \tau, t) l(x, \tau) d \tau, \\
\tilde{z}_{x}(0, t)=0, \tilde{z}(1, t)=0 .
\end{gathered}
$$

Select a positive definite Lyapunov function candidate as $V(t)=\|\tilde{z}(t)\|^{2} / 2+\left\|\tilde{z}_{x}(t)\right\|^{2} / 2$, whose first derivative of $V(t)$ becomes

$$
\dot{V}(t)=\int_{0}^{1} \tilde{z}(x, t) \tilde{z}_{t}(x, t) d x+\int_{0}^{1} \tilde{z}_{x}(x, t) \tilde{z}_{t x}(x, t) d x .
$$

By using (40) and (41) in the above equation and applying both integration by parts and Poincare inequality, we get

$$
\begin{aligned}
& \dot{V}(t)=-\varepsilon \int_{0}^{1} \tilde{z}_{x}^{2}(x, t) d x+\int_{0}^{1} d(v, x, t) \tilde{z}(x, t) d x \\
& -\int_{0}^{1} \tilde{z}(x, t) \int_{0}^{x} d(v, \eta, t) l(x, \eta) d \eta d x-\varepsilon \int_{0}^{1} \tilde{z}_{x x}^{2}(x, t) d x \\
& -\int_{0}^{1} d(v, x, t) \tilde{z}_{x x}(x, t) d x+\int_{0}^{1} \tilde{z}_{x x}(x, t) \int_{0}^{x} d(v, \eta, t) l(x, \eta) d \eta d x \\
& \leq-\varepsilon \int_{0}^{1} \tilde{z}_{x}^{2}(x, t) d x-\varepsilon \int_{0}^{1} \tilde{z}_{x x}^{2}(x, t) d x \\
& +d_{l} \int_{0}^{1}|\tilde{z}(x, t)| d x+d_{l} \int_{0}^{1}\left|\tilde{z}_{x x}(x, t)\right| d x
\end{aligned}
$$

where $d_{l}=(1+\bar{l}) \bar{d}$. By using Cauchy-Schwarz and Poincare inequalities we get

$$
\dot{V}(t) \leq-\frac{\varepsilon \pi^{2}}{8} \int_{0}^{1} \tilde{z}^{2}(x, t) d x-\frac{\varepsilon \pi^{2}}{8} \int_{0}^{1} \tilde{z}_{x}^{2}(x, t) d x+\frac{\left(\pi^{2}+4\right)}{2 \varepsilon \pi^{2}} d_{l}^{2} .
$$

Thus, $\dot{V}(t)$ will be less than zero if

$$
\|\tilde{z}(t)\|>2 d_{l} \sqrt{\pi^{2}+4} / \varepsilon \pi^{2} \text { or }\left\|\tilde{z}_{x}(t)\right\|>2 d_{l} \sqrt{\pi^{2}+4} / \varepsilon \pi^{2} .
$$

By Agmon's inequality $\max _{x \in[0,1]}|\tilde{z}(x, t)|^{2} \leq 2\|\tilde{z}(t)\|\left\|\tilde{z}_{x}(t)\right\|$, we can get $\max _{x \in[0,1]}|\tilde{z}(x, t)| \leq 2(1+\bar{l}) \bar{d} \sqrt{2\left(\pi^{2}+4\right)} / \varepsilon \pi^{2} \quad$ and $|e(t)|=|\tilde{z}(0, t)| \leq 2(1+\bar{l}) \bar{d} \sqrt{2\left(\pi^{2}+4\right)} / \varepsilon \pi^{2}$. Therefore, the detection threshold must be selected as $\rho=\rho_{0}+k_{l} \bar{d}$ where $\rho_{0}$ depends on the initial conditions and $k_{l}=2(1+\bar{l}) \sqrt{2\left(\pi^{2}+4\right)} / \varepsilon \pi^{2}$.

If an actuator fault happens $\left(\theta \neq 1, \theta_{s}=1\right)$ at $t_{i}$, then the state estimation error dynamics are given by

$$
\begin{aligned}
& \tilde{z}_{t}(x, t)=\varepsilon \tilde{z}_{x x}(x, t)+d(v, x, t)-\int_{0}^{x} d(v, \tau, t) l(x, \tau) d \tau, \\
& \tilde{z}_{x}(0, t)=0, \tilde{z}(1, t)=(\theta-1) U(t),
\end{aligned}
$$

for $t \geq t_{i}$. Now, we can define a new PDE as

$$
\begin{gathered}
\psi_{t}(x, t)=\varepsilon \psi_{x x}(x, t)+d(v, x, t)-\int_{0}^{x} d(v, \tau, t) l(x, \tau) d \tau, \\
\psi_{x}(0, t)=0, \psi(1, t)=H
\end{gathered}
$$

for $t \geq T$ and let $\psi(x, T)=\tilde{z}(x, T)$. If $|(\theta-1) U(t)| \geq H$ for $t \geq T \geq t_{i}$ then $|\tilde{z}(x, t)| \geq \psi(x, t)$ for $0 \leq x \leq 1$ and $t \geq T$. Note that the dynamics of $[\psi(x, t)-H]$ is the same as $(40-$ $41)$, thus $|\psi(0, t)-H| \leq k_{l} \bar{d}$ ultimately. Thus, for any $\omega>0$, there exists a time $t_{d} \geq T$, such that $\psi\left(0, t_{d}\right) \geq H-k_{l} \bar{d}-\omega$. That means, for any $\omega>0$, there exists a time $t_{d} \geq T$ such that $\left|\bar{e}\left(t_{d}\right)\right|=\left|\tilde{z}\left(0, t_{d}\right)\right| \geq \psi\left(0, t_{d}\right) \geq H-k_{l} \bar{d}-\omega$. Because $H>\rho+k_{l} \bar{d}$ and if $\omega$ is selected as $\omega<H-\left(\rho+k_{l} \bar{d}\right)$, then $\left|\bar{e}\left(t_{d}\right)\right|>\rho$ for some $t_{d} \geq T$, and the detection of actuator fault is guaranteed.

Since the sensor fault will affect the system through the feedback control, the detectability condition for the sensor fault is going to be very similar to that of the actuator fault. In the presence of a sensor fault $\left(\theta=1, \theta_{s} \neq 1\right)$, the detection residual is given by $\bar{e}(t)=y(t)-\hat{y}(t)=\theta_{s} z(0, t)-\hat{z}(0, t)$.

If we define a new distributed variable $\Delta$ such that $\Delta(x, t)=\theta_{s} z(x, t)-\hat{z}(x, t)$ for $0 \leq x \leq 1$, then by using (36)-(39), $\Delta$ can be described by $\Delta_{t}(x, t)=\varepsilon \Delta_{x x}(x, t)+\theta_{s}\left[d(v, x, t)-\int_{0}^{x} d(v, \tau, t) l(x, \tau) d \tau\right]$, $\Delta_{x}(0, t)=0, \Delta(1, t)=\left(\theta_{s}-1\right) U(t)$. 
and the detection residual can be defined as $\bar{e}(t)=\Delta(0, t)$. Similar to the case of actuator fault, a new bounded state variable defined by the following PDE is introduced

$$
\begin{gathered}
\Psi_{t}(x, t)=\varepsilon \Psi_{x x}(x, t)+\theta_{s}\left[d(v, x, t)-\int_{0}^{x} d(v, \tau, t) l(x, \tau) d \tau\right], \\
\Psi_{x}(0, t)=0, \Psi(1, t)=H,
\end{gathered}
$$

for $t \geq T$ and let $\Psi(x, T)=\Delta(x, T)$. If $\left|\left(\theta_{s}-1\right) U(t)\right| \geq H$ for $t \geq T$ then $|\Delta(x, t)| \geq \Psi(x, t)$ for $0 \leq x \leq 1$ and $t \geq T$. Similarly, it can be obtained that $|\Psi(0, t)-H| \leq k_{l} \bar{d} \theta_{s \max }$ ultimately, which means that for any $\omega>0$, there exists a time $t_{d} \geq T \quad$ such that $\left|\Delta\left(0, t_{d}\right)\right| \geq \Psi\left(0, t_{d}\right) \geq H-k_{l} \bar{d} \theta_{s \max }-\omega$. Therefore, by selecting $\omega<H-\left(\rho+k_{l} \bar{d} \theta_{s \max }\right)$, it is easy to see that there exists a time $t_{d} \geq T$ where $\left|\bar{e}\left(t_{d}\right)\right|=\left|\Delta\left(0, t_{d}\right)\right|>\rho$, which guarantees the detection of a fault.

Remark 7: When $d(v, x, t)=0$, observer error dynamics $\tilde{z}(x, t)$ satisfies $\tilde{z}_{t}(x, t)=\varepsilon \tilde{z}_{x x}(x, t), \tilde{z}_{x}(0, t)=0, \tilde{z}(1, t)=0$ under healthy conditions. Therefore, the observer error will converge to zero asymptotically, and the detection threshold $\rho=\rho_{0}$ only depends upon initial conditions.

\subsection{Fault Estimation}

Upon detecting a fault and knowing the fault type, an adaptive term will be incorporated into the observer defined in (39). If an actuator fault is detected, the observer in this case is described by

$$
\begin{aligned}
& \hat{z}(x, t)=\hat{\theta}(t) \Lambda(x, t)+[-\lambda /(2 \varepsilon)-q] \mathrm{A}(x, t) \\
& +\int_{0}^{1} \varepsilon\left[-l_{\eta}(s, 0)-q l(s, 0)\right] \mathrm{B}(x, s, t) d s, \quad \hat{y}(t)=\hat{z}(0, t)
\end{aligned}
$$

where $\hat{\theta}(t)$ is the estimated parameter of an actuator fault.

For a sensor fault, the observer will be described by

$$
\begin{aligned}
\hat{z}(x, t)= & \Lambda(x, t)+[-\lambda /(2 \varepsilon)-q] \mathrm{A}(x, t) / \hat{\theta}_{s}(t) \\
& +\left\{\int_{0}^{1} \varepsilon\left[-l_{\eta}(s, 0)-q l(s, 0)\right] \mathrm{B}(x, s, t) d s\right\} / \hat{\theta}_{s}(t),
\end{aligned}
$$

$\hat{y}(t)=\hat{\theta}_{s}(t) \hat{z}(0, t)$

where $\hat{\theta}_{s}(t)$ is the estimated parameter of a sensor fault. To assure $\hat{\theta}_{s}(t) \neq 0$ since $\hat{\theta}_{s}(t)$ is in the denominator in (43), two cases are considered.

Upon detecting a sensor fault, the residual becomes $\bar{e}(t)=y(t)-\hat{y}(t)=\theta_{s} z(0, t)-\hat{\theta}_{s} \hat{z}(0, t) \quad$ where $\hat{\theta}_{s}$ is initialized as $\hat{\theta}_{s}(0)=1$ and will not be updated before the detection of a sensor fault. The next theorem demonstrates the boundedness of closed-loop system with faults.

Theorem 4 (Output based fault estimation): Let the observer in (42) be used to estimate the state and output of DPS (36)-(37) with $\hat{\theta}(0)=1$. The tuning law $\dot{\hat{\theta}}=\beta \Lambda(0, t) \bar{e}(t)-\gamma \hat{\theta}$,

is activated upon detection of an actuator fault. Similarly, allow the observer from (43) to estimate the system state and output when $\hat{\theta}_{s}(0)=1$ with the tuning law

$\dot{\hat{\theta}}_{s}(t)=\left\{\begin{array}{l}0 \text { if } \beta \Lambda(0, t) \bar{e}(t)-\gamma \hat{\theta}_{s}(t)<0 \& \hat{\theta}_{s}(t)=\theta_{s \min } \\ \beta \Lambda(0, t) \bar{e}(t)-\gamma \hat{\theta}_{s}(t) \quad \text { otherwise }\end{array}\right.$

upon detection of a sensor fault, where $\theta_{\text {smin }}>0$ is a known lower bound on the sensor fault parameter. Then the residual $\bar{e}$, parameter estimation errors $\tilde{\theta}=\theta-\hat{\theta}$ and $\tilde{\theta}_{s}=\theta_{s}-\hat{\theta}_{s} \quad$ in the presence of an actuator and sensor faults respectively will be ultimately bounded (UB).

Proof: For an actuator fault, an error signal is defined as $\mu(x, t)=z(x, t)-\theta \Lambda(x, t)-(-\lambda / 2 \varepsilon-q) \mathrm{A}(x, t)$,

$$
-\int_{0}^{1} \varepsilon\left[-l_{\eta}(s, 0)-q l(s, 0)\right] \mathrm{B}(x, s, t) d s
$$

and in the case of a sensor fault, it is defined as $\mu(x, t)=z(x, t)-\Lambda(x, t)-(-\lambda / 2 \varepsilon-q) \mathrm{A}(x, t) / \theta_{s}$

$$
-\left\{\int_{0}^{1} \varepsilon\left[-l_{y}(s, 0)-q l(s, 0)\right] \mathrm{B}(x, s, t) d s\right\} / \theta_{s}
$$

This error signal in both cases clearly satisfies

$$
\begin{gathered}
\mu_{t}(x, t)=\varepsilon \mu_{x x}(x, t)+d(v, x, t)-\int_{0}^{x} d(v, \tau, t) l(x, \tau) d \tau, \\
\mu_{x}(0, t)=0, \mu(1, t)=0 .
\end{gathered}
$$

\section{(a) Actuator fault}

Now, a Lyapunov function candidate is selected as $V=\|\mu\|^{2} /(2 \varepsilon)+\tilde{\theta}^{2} /(2 \beta)$.

By taking the derivative of the Lyapunov function with respect to time and applying integration by parts, we obtain $\dot{V}=-\int_{0}^{1} \mu_{x}^{2}(x, t) d x+\int_{0}^{1} d(v(x, t), x, t) \mu(x, t) d x / \varepsilon$ $-\int_{0}^{1} \mu(x, t) \int_{0}^{x} d(v(\eta, t), \eta, t) l(x, \eta) d \eta d x / \varepsilon+\tilde{\theta}(t) \dot{\tilde{\theta}}(t) / \beta$.

Substituting (44) in the above equation yields

$$
\begin{aligned}
& \dot{V}=-\left\|\mu_{x}\right\|^{2}+\int_{0}^{1} d(v(x, t), x, t) \mu(x, t) d x / \varepsilon-\tilde{\theta}(t) \bar{e}(t) \Lambda(0, t) \\
& +\gamma \tilde{\theta}(t) \hat{\theta}(t) / \beta-\int_{0}^{1} \mu(x, t) \int_{0}^{x} d(v(\eta, t), \eta, t) l(x, \eta) d \eta d x / \varepsilon .
\end{aligned}
$$

Since $\tilde{z}(0, t)-\mu(0, t)=\tilde{\theta}(\mathrm{t}) \Lambda(0, \mathrm{t})$ and by using CauchySchwarz inequality, $\dot{V}$ can be rewritten as

$$
\begin{aligned}
\dot{V}= & -\left\|\mu_{x}\right\|^{2}+\int_{0}^{1} d(v(x, t), x, t) \mu(x, t) d x / \varepsilon \\
& -\int_{0}^{1} \mu(x, t) \int_{0}^{x} d(v(\eta, t), \eta, t) l(x, \eta) d \eta d x / \varepsilon \\
& -\bar{e}^{2}(t)+\bar{e}(t) \mu(0, t)+\gamma \tilde{\theta}(t) \hat{\theta}(t) / \beta \\
\leq & -\left\|\mu_{x}\right\|^{2}-\bar{e}^{2}(t) / 2+\mu^{2}(0, t) / 2 \\
& -\gamma\left[\tilde{\theta}^{2}(t)-\theta_{\max }^{2}\right] /(2 \beta)+d_{l} \int_{0}^{1}|\mu(x, t)| d x / \varepsilon
\end{aligned}
$$

By using the Poincare $\|\mu\|^{2} \leq 4\left\|\mu_{x}\right\|^{2} / \pi^{2}$ and Agmon's inequalities $\mu^{2}(0, t) \leq 2\|\mu\|\left\|\mu_{x}\right\| \leq\|\mu\|^{2}+\left\|\mu_{x}\right\|^{2}$, we get 


$$
\begin{aligned}
\dot{V} & \leq-\left(\pi^{2}-4\right)\|\mu\|^{2} / 8+d_{l} \int_{0}^{1}|\mu(x, t)| d x / \varepsilon-\gamma\left[\tilde{\theta}^{2}(t)-\theta_{\max }^{2}\right] /(2 \beta) \\
& \leq-\left(\pi^{2}-8\right)\|\mu\|^{2} / 8-\gamma\left[\tilde{\theta}^{2}(t)-\theta_{\max }^{2}\right] /(2 \beta)+d_{l}^{2} / 2 \varepsilon^{2}
\end{aligned}
$$

Therefore, $\dot{V}<0$ when

$$
\|\mu\|>\sqrt{\frac{4\left(\gamma \theta_{\max }^{2}+\beta d_{l}^{2} / \varepsilon^{2}\right)}{\left(\pi^{2}-8\right) \beta}} \text { or }|\tilde{\theta}|>\sqrt{\frac{\gamma \theta_{\max }^{2}+\beta d_{l}^{2} / \varepsilon^{2}}{\gamma}} \text {. }
$$

Hence, $\mu$ and $\tilde{\theta}$ are UB with the bounds defined above. Since $\bar{e}(t)=\mu(0, t)+\tilde{\theta}(t) \Lambda(0, t)$ and $\Lambda$ is bounded, $\bar{e}$ is also bounded.

Remark 8: In the ideal case, when $d(v, x, t)=0$, one can show that $\|\mu\| \leq \sqrt{4 \gamma /\left[\left(\pi^{2}-4\right) \beta\right]} \theta_{\max }$ and $|\tilde{\theta}| \leq \theta_{\max }$.

\section{(b) Sensor fault}

Similarly, in this case, consider the Lyapunov function

$$
V=\|\mu\|^{2} /(2 \varepsilon)+\tilde{\theta}_{s}^{2} /\left(2 \beta \theta_{s \max }^{2}\right)
$$

where $\theta_{\text {smax }}$ is the upper bound on the sensor fault magnitude $\theta_{s}$. Taking the derivative of (46) with respect to time and applying integration by parts leads to

$$
\dot{V}=-\int_{0}^{1} \mu_{x}^{2}(x, t) d x+\int_{0}^{1} d(v(x, t), x, t) \mu(x, t) d x / \varepsilon
$$$$
-\frac{1}{\varepsilon} \int_{0}^{1} \mu(x, t) \int_{0}^{x} d(v(\eta, t), \eta, t) l(x, \eta) d \eta d x+\frac{\theta_{s}(t) \dot{\tilde{\theta}}_{s}(t)}{\beta \theta_{s \max }^{2}} .
$$

The sensor fault parameter $\hat{\theta}_{s}$ is tuned using $\dot{\hat{\theta}}_{s}(t)=\beta \Lambda(0, t) \bar{e}(t)-\gamma \hat{\theta}_{s}(t)$. However, $\hat{\theta}_{s}$ appears in the denominator of (43), and the update law is modified as (45) to ensure it is nonzero. With this update law, if $\beta \Lambda(0, t) \bar{e}(t)-\gamma \hat{\theta}_{s}(t)<0 \& \hat{\theta}_{s}=\theta_{s \min }, \dot{V}$ is given by $\dot{V} \leq-\left\|\mu_{x}\right\|^{2}+d_{l} \int_{0}^{1}|\mu(x, t)| d x / \varepsilon$; otherwise, it should satisfy $\dot{V} \leq-\left\|\mu_{x}\right\|^{2}-\frac{\tilde{\theta}_{s} \Lambda(0, t) \bar{e}(t)}{\theta_{s \max }^{2}}+\frac{\gamma \tilde{\theta}_{s}(t) \hat{\theta}_{s}(t)}{\beta \theta_{s \max }^{2}}+\frac{d_{l}}{\varepsilon} \int_{0}^{1}|\mu(x, t)| d x$. In the first case, by applying Poincare inequality $\|\mu\|^{2} \leq \frac{4\left\|\mu_{x}\right\|^{2}}{\pi^{2}}$, we can show $\dot{V} \leq-\frac{\left(\pi^{2}-2\right)}{4}\|\mu\|^{2}+\frac{d_{l}^{2}}{2 \varepsilon^{2}}$ which means $\dot{V}$ will be less than zero if $\|\mu\|>\sqrt{2 /\left[\varepsilon^{2}\left(\pi^{2}-2\right)\right]} d_{l}$. Therefore, $\|\mu\|$ and $\left|\tilde{\theta}_{s}\right|$ are bounded. Now for the second case, as $\bar{e}(t)-\theta_{s} \mu(0, t)=\tilde{\theta}_{s}(t) \Lambda(0, t), \dot{V}$ can be written as

$$
\begin{aligned}
\dot{V} \leq & -\left\|\mu_{x}\right\|^{2}-\left[\bar{e}(t)-\theta_{s} \mu(0, t)\right] \bar{e}(t) / \theta_{s \max }^{2} \\
& +\gamma \tilde{\theta}_{s}(t) \hat{\theta}_{s}(t) /\left(\beta \theta_{s \max }^{2}\right)+d_{l} \int_{0}^{1}|\mu(x, t)| d x / \varepsilon \\
\leq & -\left\|\mu_{x}\right\|^{2}+d_{l} \int_{0}^{1}|\mu(x, t)| d x / \varepsilon+\mu^{2}(0, t) / 2 \\
& -\gamma \tilde{\theta}_{s}^{2}(t) /\left(2 \beta \theta_{s \max }^{2}\right)+\gamma /(2 \beta)
\end{aligned}
$$

Applying Poincare $\|\mu\|^{2} \leq 4\left\|\mu_{x}\right\|^{2} / \pi^{2}$ and Agmon's inequalities $\mu^{2}(0, t) \leq 2\|\mu\|\left\|\mu_{x}\right\| \leq\|\mu\|^{2}+\left\|\mu_{x}\right\|^{2}$ will lead to

$$
\begin{aligned}
& \dot{V} \leq-\left(\pi^{2}-4\right)\|\mu\|^{2} / 8+d_{l} \int_{0}^{1}|\mu(x, t)| d x / \varepsilon \\
& -\gamma \tilde{\theta}_{s}^{2}(t) /\left(2 \beta \theta_{s \max }^{2}\right)+\gamma /(2 \beta) \\
& \leq-\left(\pi^{2}-8\right)\|\mu\|^{2} / 8-\gamma \tilde{\theta}_{s}^{2}(t) / 2 \beta \theta_{s \max }^{2}+\gamma / 2 \beta+d_{l}^{2} / 2 \varepsilon^{2}
\end{aligned}
$$

Thus, $\dot{V}<0$ when

$\|\mu\|>\sqrt{\frac{4\left(\gamma+\beta d_{l}^{2} / \varepsilon^{2}\right)}{\left(\pi^{2}-8\right) \beta}}$ or $\left|\tilde{\theta}_{s}\right|>\sqrt{\frac{\gamma+\beta d_{l}^{2} / \varepsilon^{2}}{\gamma}} \theta_{s \max }$,

are satisfied, we can see how $\mu$ and $\tilde{\theta}_{s}$ are UB. Because $\bar{e}(t)=\theta_{s} \mu(0, t)+\tilde{\theta}_{s}(t) \Lambda(0, t) \quad$ and $\theta_{s}, \mu, \tilde{\theta}_{s}$ and $\Lambda$ are bounded, $\bar{e}$ is also bounded. Therefore, the closed-loop system is bounded for both cases.

Remark 9: Without disturbance one can show that $\|\mu\| \leq \sqrt{4 \gamma /\left[\left(\pi^{2}-4\right) \beta\right]}$ and $|\tilde{\theta}| \leq \theta_{s \max }$.

\subsection{Failure Prediction}

A system may remain functional after an incipient fault; however, it cannot function after a failure. The TTF scheme can predict the RUL of the system upon detection by using the estimated parameter trajectories. The TTF can be defined as the difference between current time $t$ and the time of failure $t_{f} \operatorname{TTF}(t)=t_{f}-t$. The TTF can be predicted by using the parameter update laws (44) and (45) given the parameter failure limits as proposed next.

Remark 10 (TTF prediction for an actuator fault): Given the detection residual of an actuator fault, the input filter state and the upper bound of the actuator fault parameter, upon detecting an actuator fault by the observer (39), TTF can then be estimated as

$$
\operatorname{TTF}(t)=\frac{1}{\gamma} \operatorname{Ln}\left(\frac{\gamma \hat{\theta}(t)-\beta \Lambda(0, t) \bar{e}(t)}{\gamma \theta_{f}-\beta \Lambda(0, t) \bar{e}(t)}\right),
$$

where $\theta_{f}$ is the failure limit for $\theta$. Considering $\beta \Lambda(0, t) \bar{e}(t)$ as an input to (44), the fault parameter estimation can be solved as

$$
\hat{\theta}\left(t_{f}\right)=e^{-\gamma\left(t_{f}-t\right)} \hat{\theta}(t)+\int_{t}^{t_{f}} e^{-\gamma\left(t_{f}-\tau\right)} \beta \Lambda(0, \tau) \bar{e}(\tau) d \tau \text { for } t_{f}>t
$$

where $t$ is the current time instant and $t_{f}$ refers to future time. Now assume that the term $\beta \Lambda(0, t) \bar{e}(t)$ is held in the interval $\left[t, t_{f}\right]$ and let $t_{f}$ be the first time when the value of $\hat{\theta}$ reaches its failure limit $\theta_{f}$ as $\hat{\theta}\left(t_{f}\right)=\theta_{f}=e^{-\gamma\left(t_{f}-t\right)} \hat{\theta}(t)+\beta \Lambda(0, t) \bar{e}(t)\left[1-e^{-\gamma\left(t_{f}-t\right)}\right] / \gamma$.

By substituting $T T F=t_{f}-t$ in the above equation and solving it, we will get (47).

Remark 11 (TTF prediction for a sensor fault): Given the detection residual of a sensor fault, input filter state and the upper bound of the sensor fault parameter, upon detecting a sensor fault by the observer (39), the TTF can be estimated as 
$\operatorname{TTF}(t)=\frac{1}{\gamma} \operatorname{Ln}\left(\frac{\gamma \hat{\theta}_{s}(t)-\beta \Lambda(0, t) \bar{e}(t)}{\gamma \theta_{s_{f}}-\beta \Lambda(0, t) \bar{e}(t)}\right)$,

where $\theta_{s f}$ is the failure limit for $\theta_{s}$. Similarly, for the sensor fault, assume that in the interval $\left[t, t_{f}\right]$, the term $\beta \Lambda(0, t) \bar{e}(t)$ is held and suppose $t_{f}$ is the first time when the value of $\hat{\theta}_{s}$ reaches its failure limit $\theta_{s_{f}}$; hence, the estimated TTF is given by (48).

\section{Simulation Results}

Consider a thin rod whose heat conduction can be represented by parabolic PDEs. The heat equation with an actuator fault can be expressed as

$v_{t}(x, t)=\varepsilon v_{x x}(x, t)+\lambda v(x, t)+d(v(x, t), x, t)$,

$v_{x}(0, t)=-q v(0, t), \quad v(1, t)=\theta U(t)$,

where $d(x, t)=0.05 e^{-0.5(x-0.2)^{2}} \sin (t)$ is the disturbance. The system state $v(x, t)$ represents the temperature in this heated rod with $v_{d}(0, t)=5\left(1-e^{0.5 t}\right)$ being the desired output temperature profile. In addition, $U(t)=v_{d}(1, t)+\int_{0}^{1} k(x)\left[v(x, t)-v_{d}(x, t)\right] d x$ is the control input designed to regulate the temperature profile of the heated rod with $k(x)=-\frac{\lambda}{2} \sum_{n=0}^{50} \frac{\left[\lambda\left(1-x^{2}\right)\right]^{n}}{4^{n} n !(n+1) !}$ being the controller gain,

and $v_{d}(x, t)=5\left[\cos (\sqrt{\lambda} x)-e^{-0.5 t} \cos (\sqrt{0.5+\lambda} x)\right]$ being the desired full state trajectory. Notice that in the output measurement case, $\hat{v}(x, t)$ will be utilized instead of $v(x, t)$ resulting in $U(t)=v_{d}(1, t)+\int_{0}^{1} k(x)\left[\hat{v}(x, t)-v_{d}(x, t)\right] d x$.

The system parameters chosen for the simulation are given by $\varepsilon=1$, and $q=0$. Now $\theta=1-.5 \Omega(t-5)$ is the actuator fault $\lambda=10$ parameter seeded at $t=5 \mathrm{~s}$ where $\Omega(t-5)=\left\{\begin{array}{ll}0 & \text { if } t<5 \\ 1-e^{-0.3(t-5)}, & \text { if } t \geq 5\end{array}\right.$.

For the numerical simulation, the closed-loop system and observer are discretized by using the finite difference method with 20 grid points with respect to the space. The total time for simulation is taken as 25 seconds. The number of discretization points should be selected carefully since it may result in inaccurate results for the PDE system. Simulation results for full state measurement case can be found in research reported by Cai et al. (2014). Here, results are obtained with the output temperature at $x=0$ as measured.

\subsection{Actuator Fault}

The initial condition of actual system (49)-(50) is selected as $v(x, 0)=0.2 \cos (x)$, and the initial values for the filters are set at zero. The estimated fault parameters are initialized as $\hat{\theta}(0)=1, \theta_{s}(0)=1$, and the threshold is selected as $\rho=0.5$. First, by applying the transformation (35), the DPSs (49) and (50) are converted to the observable form in (36) and (37) where $l_{\eta}(x, 0)=25(1-x) \sum_{n=0}^{50} \frac{(-1)^{n}[10 x(2-x)]^{n}}{4^{n} n !(n+2) !}$ and $q=0$. Then two output filters along with one input filter are employed to estimate the state and output of the transformed system. Prior to the fault occurrence, the detection residual is expected to decrease, whereas it will increase once a fault occurs. This is clearly observed in Fig. 2. Fault is detected at approximately $t=6.3 \mathrm{~s}$ when the detection residual exceeds the threshold.

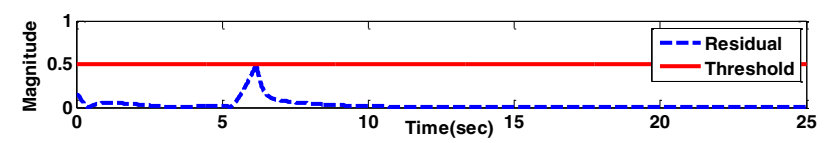

Fig. 2. Detection residual of actuator fault.
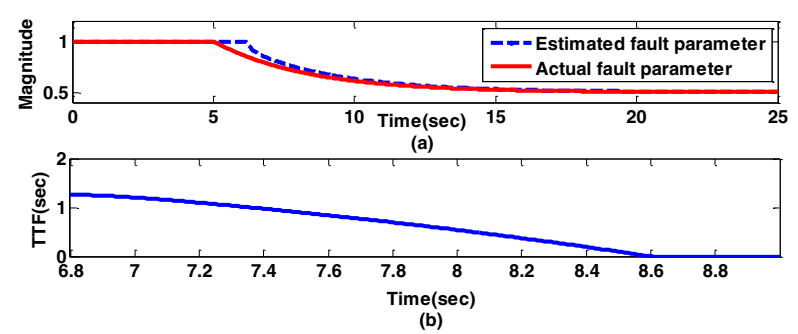

Fig. 3 (a) Actual and estimated fault; (b) estimated TTF.

Upon detection of the actuator fault, the adaptive estimator will be utilized to learn the fault dynamics. In this case, we just have one available measurement, so the update law (44) is utilized to estimate the actuator fault parameter where update parameters are chosen as $\beta=0.2$ and $\gamma=0.01$. The fault estimation results can be seen from Fig. 3(a). According to the estimated fault parameter and the failure limit of fault parameter defined as $\theta_{f}=0.7$, the estimated TTF is obtained by using the formula (47). Estimated TTF is plotted in Fig. 3(b).

\subsection{Sensor Fault}

As discussed in Section 4, this method is able to deal with a sensor fault as well. Thus, a sensor fault is expressed as $y=\theta_{s} v(0, t)$ with the fault function being described by $\theta_{s}(t)=1+\Omega(t-5) \quad$ where $\Omega(t)=\left\{\begin{array}{ll}0 & , \text { if } t<5 \\ 1-e^{-0.3(t-5)} & \text {, if } t \geq 5\end{array}\right.$ and the disturbance is selected as $d(x, t)=0.03 e^{-0.5(x-0.3)^{2}} \sin (2 t)$. The detection observer in (39) is again used to detect the fault. Fig. 4 depicts the detection residual as exceeding the threshold around one and half seconds after the initiation of the fault. Upon detecting the sensor fault, the adaptive terms are ac- 
tivated in the observer as described in (43). By using the parameter tuning law given by Theorem 4 with the parameters selected as $\beta=0.35$ and $\gamma=0.01$, the fault parameter estimation can be performed, the TTF can be estimated by (48), and the failure limit utilized in the formula is $\theta_{s f}=1.5$. Fault estimation and TTF prediction results are shown in Fig. 5.

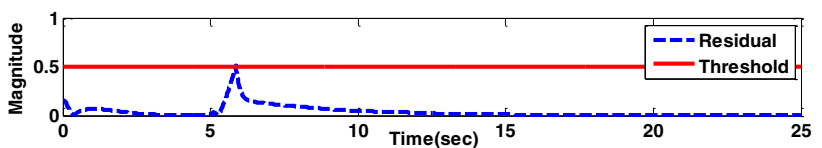

Fig. 4. Detection residual of a sensor fault.
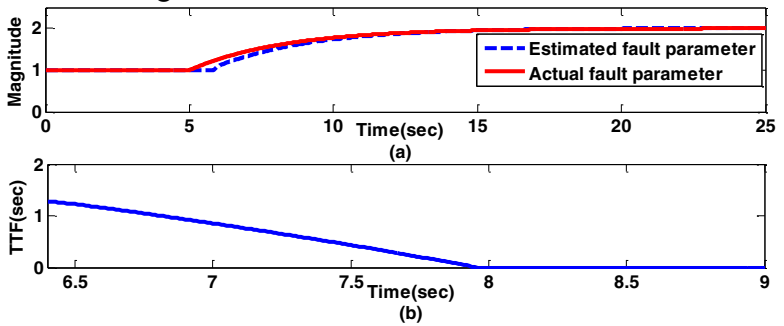

Fig. 5. (a) Actual and estimated fault dynamics and (b) TTF.

\section{Conclusions}

A novel observer based on the PDE representation of a DPS provides a more accurate estimation of the state which is beneficial to both fault detection and estimation. The adaptive term incorporated in the observer appears to estimate the fault function. The TTF can be predicted based on both estimated fault parameters and a failure threshold provided the fault type is known.

The filter-based approach is quite important when dealing with implementation of the scheme on real practical systems, and it also allows the estimation of actuator and sensor faults provided the fault type is given. Simulation results confirm the theoretical claims. Future research will involve fault isolation and extension to other PDEs.

\section{References}

Balogh, A., and Krstic, M. (2002). Infinite dimensional backstepping-style feedback transformations for a heat equation with an arbitrary level of instability. European journal of control, $8(2), 165-175$

Baniamerian, A., and Khorasani, K. (2012, June). Fault detection and isolation of dissipative parabolic PDEs: Finitedimensional geometric approach. In American Control Conference (ACC), 2012 (pp. 5894-5899).

Bartelmus, W. (2001). Mathematical modeling and computer simulations as an aid to gearbox diagnostics. Mechanical Systems and Signal Processing, 15(5), 855-871.

Biswas, G., Koutsoukos, X., Bregon, A., and Pulido, B. (2009, June). Analytic redundancy, possible conflicts, and TCGbased fault signature diagnosis applied to nonlinear dynamic systems. In Proc. of the 7th IFAC Symp. on Fault Detection, Supervision and Safety of Tech. Processes (pp. 1486-1491).

Cai, J., Ferdowsi, H., and Jagannathan, S. (2014, December). Model-based fault diagnosis and prediction for a class of distributed parameter systems. Decision and Control (CDC), IEEE 53rd Annual Conference on (pp. 5758-5763).
Chinnam, R. B., and Baruah, P. (2003). Autonomous diagnostics and prognostics through competitive learning driven HMMbased cluster. Proc. of the International Joint Conference on Neural Networks, New York, US (pp. 2466-2471).

Christofides, P. D. (2001). Control of nonlinear distributed process systems: recent developments and challenges. AIChE Journal, 47(3), pp. 514-518.

Demetriiou, M. A., and Ito, K. (2002). On-line fault detection and diagnosis for a class of positive real infinite dimensional systems. Proceedings of the 41st IEEE Conference on Decision and Control, Las Vegas, US (pp. 4359-4364).

Gertler, J. (2005). Residual generation from principal component models for fault diagnosis in linear systems. Proc. of the IEEE International Sym. on Intelligent Control, Cyprus (pp. 634-639).

Hansen, R. J., Hall, D. L., and Kurtz, S. K. (1995). A new approach to the challenge of machinery prognostics. Journal of Engineering for Gas Turbines and Power, 117(2), 320-325.

Hardy, G. H., Littlewood, J. E., and Polya, G. (1952). Inequalities. Cambridge: Cambridge university press.

Isermann, R. (2004). Model-based fault-detection and diagnosisstatus and applications. Annual Reviews in Ctrl, 29(1), 71-85.

Jiang, B., Chowdhury, F. N. (2005). Parameter fault detection and estimation of a class of nonlinear systems using observers. Journal of the Franklin Institute, 342(7), 725-736.

Jiang, B., Marcel, S., and Vincent, C. (2006). Fault accommodation for nonlinear dynamic systems. IEEE Transaction on Automatic Control, 51(9), 1578-1583.

Krstic, M., and Smyshlyaev, A. (2008). Boundary control of PDEs. Philadelphia: Society for Industrial and Applied Mathematics.

Kwan, C., Zhang, X., Xu, R., and Haynes, L. (2003). A novel approach to fault diagnostics and prognostics. Proc. of the IEEE Int. Conf. on Robotics and Auto, NY (pp. 604-609).

LaSalle, J. and Lefschetz, S. (1961). Stability by Lyapunov's Direct Method with Applications, Academic Press, New York.

Meseguer, J., Puig, V., and Escobet, T. (2006, August). Observer gain effect in linear interval observer-based fault detection. In Fault Detection, Supervision and Safety of Technical Processes (Vol. 6, No. 1, pp. 540-545).

Patan, M, and Ucinski, D. (2005). Optimal activation strategy of discrete scanning sensors for fault detection in distributed parameter systems. Proceedings of the 16th IFAC world congress, Prague, Czech Republic (pp. 1836-1836).

Smyshlyaev, A., and Krstic, M. (2004). Backstepping observer for a class of parabolic PDEs. System and Control Letters, 54(7), 613-625.

Smyshlyaev, A., and Krstic, M. (2010). Adaptive control of parabolic PDEs. New Jersey: Princeton University Press.

Vania, A, and Pennacchi, P. (2004). Experimental and theoretical application of fault identification measures of accuracy in rotating machine diagnostics. Mechanical Systems and Signal Processing, 18(2), 329-352.

Wang, P., and Vachtsevanos, G. (2001). Fault prognostics using dynamic wavelet neural networks. AI EDAM-Artificial Intelligence for Engineering Design Analysis and Manufacturing, 15(4), pp. 349-365.

Yao, Z., and El-Farra, N. H. (2011, December). Robust fault detection and reconfiguration in sampled-data uncertain distributed processes. In Decision and Control and European Control Conference (CDC-ECC), 50th IEEE Conference on (pp. 4925-4930). 